\title{
IoT based Real-time Air Quality Monitoring and Control System to Improve the Health and Safety of Industrial Workers
}

\author{
T.Veeramanikandasamy, Gokul Raj.S, A.Balamurugan, A.P.Ramesh, Y.A.Syed Khadar
}

\begin{abstract}
Pollution is adding contaminants into the nature that causes an adverse change in the environment. Air pollution is one of the highest mortality risk factors globally. The sources of air pollution in the industries are power plants, chamber process, cleaning, burning of materials, etc. A variety of pollutants emitted into the air such as sulfur dioxide, carbon monoxide, carbon dioxide, ammonia and volatile organic compounds. Particulate Matter (PM) is an air pollutant that is the mixture of solid dust or pollen and liquid droplets with air. Air pollution in industrial workplaces is a major concern and monitoring and management of it to be addressed to protect the industrial workers health from the air pollution effects. The people are suffering from several respiratory and heart issues along with cancer due to increasing air pollution. This device is composed of ESP32 MCU, MQ135 gas sensor, SDS011 optical dust particle sensor, and BME280 humidity and temperature sensor for monitoring the air quality. The gas sensor MQ135 senses the harmful gases present in the environment. SDS011 optical dust sensor senses the PM levels $\left(P M_{10}\right.$ and $\left.P M_{2.5}\right)$ in the atmosphere. The sensor values are evaluated for the Air Quality Index (AQI) and display it on the ThingSpeak IoT platform. Vrituino app has used for a virtual screen with widgets on the mobile phone to monitor the system using the web. In order to improve the real-time performance of the system, an IoT and a cloud computing technology are being used. The ESP32 turns on the fan units to maintain the pollutants within the safe limit when the presence of harmful gases and PM levels exceeds a certain threshold level. This system is essential for industrial work places to adopt measures and control air pollution which increase industrial workers safety.
\end{abstract}

Keywords: Air Pollution, Particulate Matter, ESP32, IoT, Air Quality Index (AQI), ThinkSpeak, Vrituino.

Revised Manuscript Received on February 28, 2020.

* Correspondence Author

T.Veeramanikandasamy*, Department of Electronics and Communication Systems, Sri Krishna Arts and Science College, Coimbatore 641008, Tamilnadu, India. E-mail: veeramaniks@gmail.com.

Gokul Raj.S, Department of Electronics and Communication Systems, Sri Krishna Arts and Science College, Coimbatore 641008, Tamilnadu, India.

A.Balamurugan, Department of Physics, Government Arts and Science College, Avinashi 641654, Tamilnadu, India. E-mail: bala.snr@gmail.com.

A.P.Ramesh, Department of Electronics and Communication Systems, Sri Ramakrishna College of Arts and Science, Coimbatore 641006, Tamilnadu, India.

Y.A.Syed Khadar, Department of Physics, K.S.R College of Arts and Science for Women, Tiruchengode 697215, Tamilnadu, India.

(C) The Authors. Published by Blue Eyes Intelligence Engineering and Sciences Publication (BEIESP). This is an open access article under the CC-BY-NC-ND license http://creativecommons.org/licenses/by-nc-nd/4.0/

\section{INTRODUCTION}

Considering the daily newspapers and other electronic or print media, they show the devastating news about people are becoming sick and the climate is changing such a way that it becomes miserable for living people.

All people are suffering from the curse of climate change. The main reason for people's health issues and climate change is air pollution. The air pollution has brought changes in climate like global warming, global dimming, over raining, drought, storms, acid rain, foggy weather, etc. In recent days, the various pollutions like water pollution, soil contamination, noise pollution, radioactive contamination, plastic pollution, thermal pollution, and other pollution are affecting both human health and cause environmental problems. Although air pollution has the highest priority compared to others because air is the most useful for every living thing. The living things on earth are suffering from bronchitis, heart diseases, pneumonia, lung and aggravated asthma due to insufficient air quality.

The air quality has to be maintained for protecting human health, the World Health Organization (WHO) provides the threshold pollutant concentration levels to limit harmful effects on human mortality. The daily and annual limits of fine $\mathrm{PM}\left(\mathrm{PM}_{2.5}\right.$, which has a diameter less than or equal to 2.5 $\mu \mathrm{m})$ do not exceed 25 and $50 \mu \mathrm{g} / \mathrm{m}^{3}$, respectively. However, there is no specific recommendation for safe PM limits concerning cognition and mental health [1]. The Lancet Planetary Health journal declared that with eighteen percent of the global population, India suffered twenty six percent of premature mortality and health loss attributable to air pollution globally. In 2017, half of the deaths (aged less than 70 ) in India due to air pollution. It asserted that seventy seven percent of India's population is exposed to above NAAQS safe limit of outdoor air pollution levels.

The research is going on in this serious issue to monitor and control the quality of air. The main purpose of this system is to estimate the quality of air for people and any other living thing which exists on the earth [2]. The proposed embedded system model includes various sensors like MQ135 (sensitive to $\mathrm{CO}_{2}, \mathrm{NO}_{\mathrm{x}}, \mathrm{SO}_{2}, \mathrm{NH}_{3}$, and benzene), BME280 (Humidity and Temperature sensor) and SDS011 (Optical dust particle sensor) and a dust collector. The system can monitor the gases, toxic level of Particulate Matter (PM), 
and air quality index (AQI) of the industrial workplaces and display it on the IoT ThingSpeak dashboard. The real-time system can turn on the dust collector/fan units when pollution goes beyond the level and send the alert message to the control room authorities.

This will lead to early gas leak detection which prevents major accidents in the industry. Recently, most of the major cities in the world are polluted.

Therefore, this system can also be installed anywhere and connected with a cloud network to monitor the level of the AQI category.

\section{MOTIVATION FOR THE PROPOSED WORK}

There are three things that a human needs for a healthy life. They are air, water, and food. But recently the air is polluted more as compared to water and food.

Air pollution causes premature death of human being and it is a significant risk factor for the number of pollution-related diseases including heart disease, respiratory infections, stroke and lung cancer [3].

The health problem caused by air pollution includes coughing, difficulty in breathing, wheezing, asthma, and worsening of existing respiratory and cardiac conditions. These may result in increased medication use, more hospital admissions and premature death.

Thus, air pollution is an important problem which is faced by all countries in the world. It must be reduced as much as possible.

There are more pollutions e.g. water pollution, noise pollution, plastic pollution, soil contamination but air pollution is the most alarming issue and this should be studied for the sake of saving the world.

The most open-air pollution sources include industries, vehicles, power generation, building heating systems, solid waste, and agriculture incineration.

Besides, more than four billion people in the world rely on polluting technologies and discharge pollutants to outdoors from fuels (including biomass, coal) for household cooking, and lighting, etc.

It basically figured out the emission rate of poisonous gasses which are responsible for air pollution. The low-cost air quality system was discussed in 2008 because at that time the sensors were quite expensive and also the system [4]. The industrial air pollution monitoring system for safety and health enhancement was introduced to know the hazardous gasses and their impact.

By using the mobile GPRS system the air pollution information could be transmitted. Wireless sensor network-based pollution monitoring system in metropolitan cities was introduced to know the air quality.

The pollution Dynamic Monitoring System is also done previously. We need a smart environment monitoring system with IoT support to know the information on the mobile itself.

\section{HARDWARE IMPLEMENTATION}

The proposed model for the IoT based air quality monitor and control using an embedded system is shown in Fig. 1.

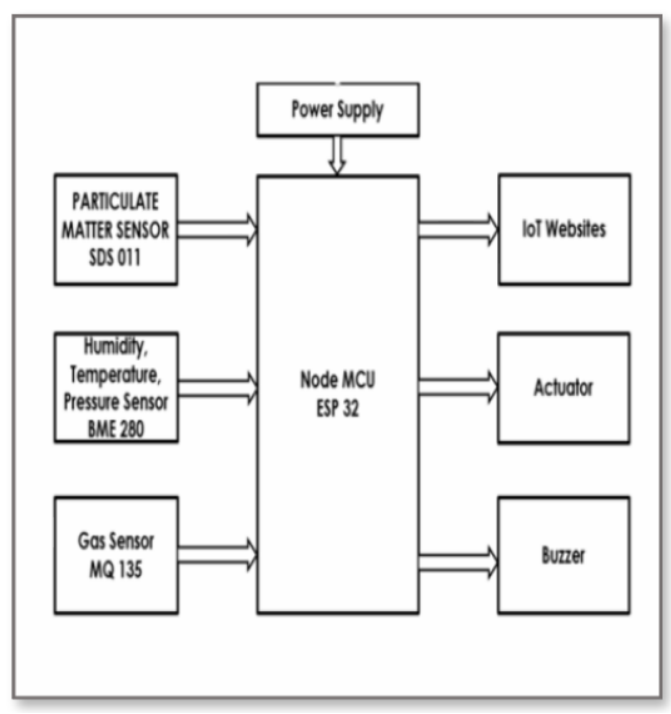

Fig. 1. The proposed model for the IoT based air quality monitor and control using embedded system.

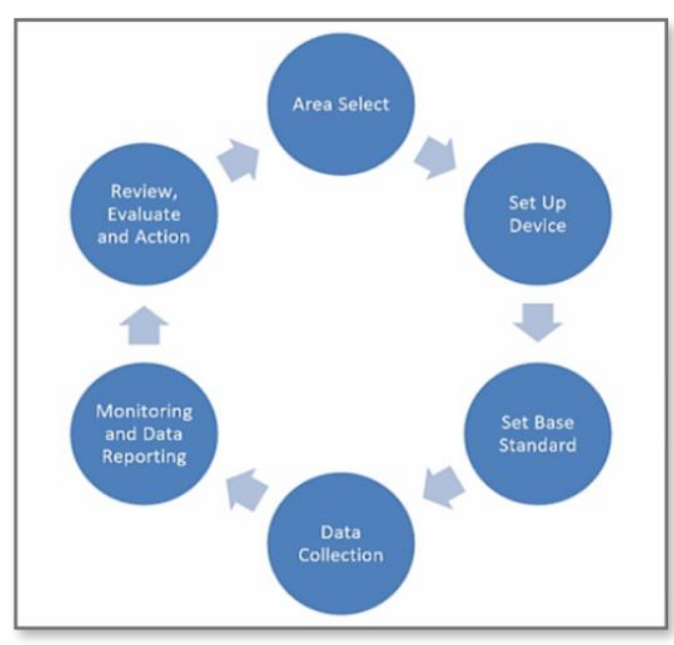

Fig. 2.Sequence of the system process.

The device is set up to collect the environmental data through various sensors and these data are acts as an input to the program sequence to update the output. The sensors receive analog data from the environment which is converted into digital by itself and then sent to the server with the help of the ESP32 module where all the data are stored in the cloud [5].

The system process can be done based on the sequence given in figure 2 . The system analyzes the air quality in real-time and log data to a remote server, and maintains the data updating over the internet.

Air quality measurements have taken based on the ppm (Parts per Million) metrics and analyzed. The result is displayed on the display interface and could be accessed via the cloud on smart mobile devices using Virtuino. If the threshold values for pollution levels are exceeded, the application sends notifications to the users.

The users able to monitor the measured parameters on their smart phones. The air quality measurements are sent to the cloud server in every one-minute intervals. The figure 3 shows the list of components used in the proposed system. 


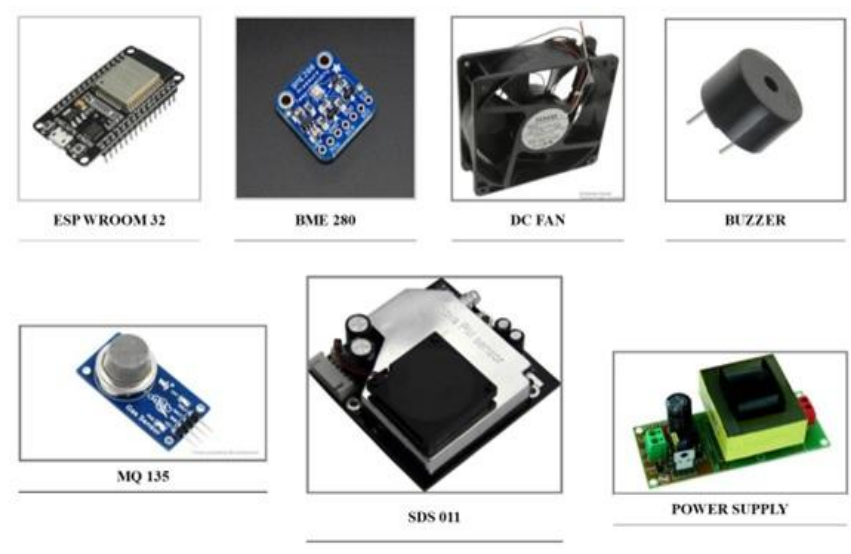

Fig. 3.Components of the proposed system.

\section{A. Node MCU (ESP32)}

ESP32 is a series of low-power and low-cost SoC with integrated Wi-Fi and Bluetooth microcontroller module for smart system implementation and IoT applications. The ESP32 MCU employs a Harvard Tensilica Xtensa LX6 32-bit dual-core processor capable of operating frequency up to $240 \mathrm{MHz}$ and includes in-built low-noise receive amplifier, antenna switches, power amplifier, powerful CPU, power-management modules, and filters [6].

\section{B. Environmental Sensor BME280}

The BME280 is an integrated environmental MEMS sensor that is specifically developed for mobile applications by Bosch Sensortec. The size, low power consumption and operating temperature of about -40 to $85{ }^{\circ} \mathrm{C}$ are key design features. This unit has high linearity and high accuracy to measure pressure, humidity, and temperature with the help of I2C and SPI communication protocol. It is designed for low current consumption $(1.8 \mu \mathrm{A})$ with average current consumption in sleep mode is $0.1 \mu \mathrm{A}$, high stability with multi-function, and high EMC robustness. BME280 is housed in a compact 8-Pin LGA with a metal package and a footprint of only $2.5 \times 2.5 \mathrm{~mm}^{2}$ with a height of 0.93 millimeters. The figure 4 shows humidity in percentage and temperature in degree Celsius readings from environmental sensor BME280.
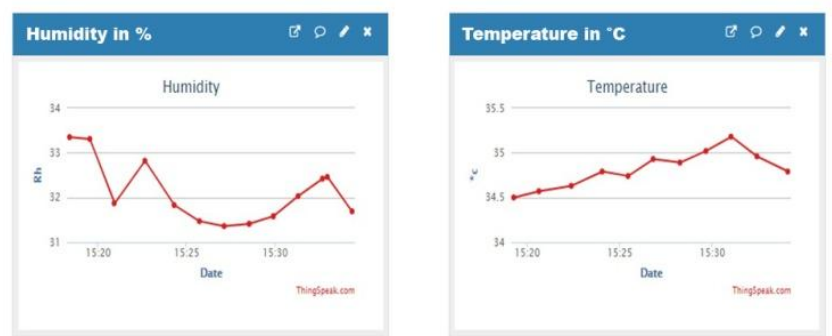

Fig. 4.Humidity and Temperature Charts.

\section{Gas Sensor MQ135}

The MQ135 gas sensor senses gases like oxygen, alcohols, ammonia nitrogen, sulfide, aromatic compounds and smoke. This gas sensor utilizes a small heater inside with an electrochemical sensor and it is sensitive to a range of gasses in an environment at room temperature [7]. MQ135 sensor has lower conductivity in clean air. When the target exposé to gas, the sensor conductivity increases with increasing the gas concentration. An electronic circuit is used to convert the change of gas concentration to the corresponding conductivity output. This sensor used to sense the presence of gases like $\mathrm{NH}_{3}$, sulphide, benzene, and $\mathrm{CO}_{2}$. The figure 5 shows the ppm values of $\mathrm{CO}_{2}$ from gas sensor MQ135. If the ppm value exceeds 450, the MCU produced sound using buzzer and turn on the exhaust fan.

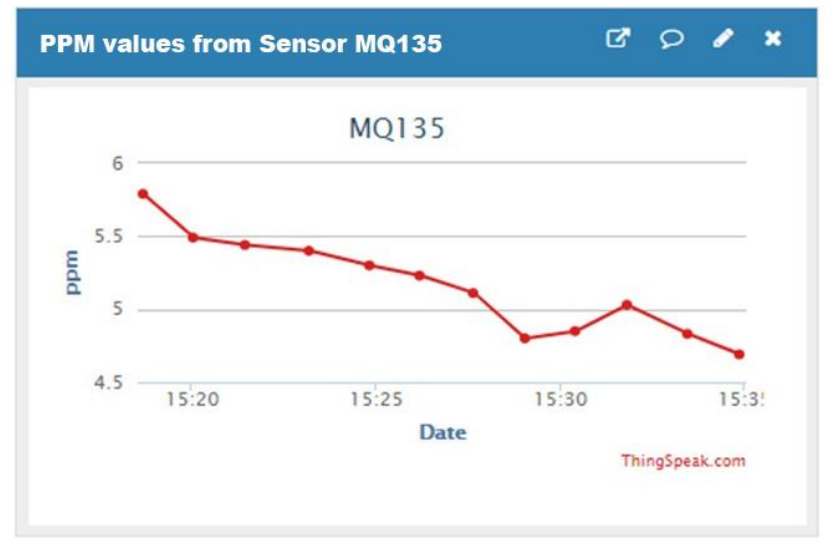

Fig. 5.The char for ppm values from gas sensor MQ135.

\section{Buzzer}

Typical uses of buzzer and beeper or alarm device confirm the inputs and device outputs by making sound. It may be mechanical, piezoelectric and electromechanical component.

\section{E. Particulate Matter Sensor SDS011}

The SDS 011 Sensor is a quite recent Air Quality Sensor developed by Inova fit, University of Jinan. The technology used for the measurement is based on laser diffraction theory where particle density distribution is counted from the light intensity distribution patterns [8]. The sensor output is digital that can be used to measure the particle distribution density between 0.3 to $10 \mu \mathrm{m}$ in the air. SDS 011 Sensor has a built-in algorithm that converts the particle density distribution into particle mass. It is used to measure the concentration of $\mathrm{PM}_{2.5}$ and $\mathrm{PM}_{10}$ in air. The figure 6 shows the readings from PM sensor SDS011. The $\mathrm{PM}_{2.5}$ and $\mathrm{PM}_{10}$ values are plotted in $\mu \mathrm{g} / \mathrm{m}^{3}$. If the $\mathrm{PM}_{10}$ value exceeds 200 , the MCU will turn on the actuator of dust collector. Similarly if the $\mathrm{PM}_{2.5}$ value exceeds 50, the MCU turn on actuator of dust collector.
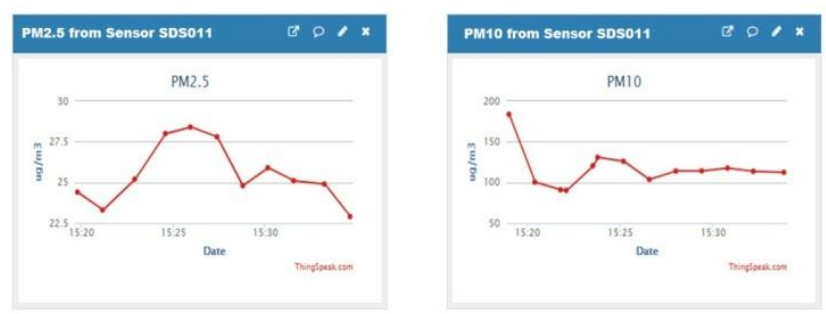

Fig. 6. The chars for $P M_{2.5}$ and $\mathrm{PM}_{10}$ values of an air from SDS011.

\section{F. Actuator with Dust Collector}

The dust collector system needs a motor and a fan which supplies mechanical energy to move the contaminated air from the dust-producing sources. The dust collector has the filtration system for the fine removal. 


\section{IoT based Real-time Air Quality Monitoring and Control System to Improve the Health and Safety of Industrial Workers}

\section{SOFTWARE IMPLEMENTATION FOR IOT}

This project uses the concept of IoT for monitoring and controlling the system using a ThingSpeak cloud server. The Node MCU is configured to transfer the measured data of all sensors on a remote server. The online application provides global access to the measured data using any device that has internet connection capability. Data collected from the sensors are analyzed and passed the information in the form of a string to update the web page in the remote server. The system also uses an android app called Virtuino which shows the sensors data graphically. In this app, one has to subscribe to a topic and publish a message of the specific function. Virtuino allows sending the SMS when the sensor reading crosses a threshold.

\section{A. ThingSpeak}

ThingSpeak is an IoT analytics platform service that allows aggregating, visualizing and analyzing live data streams in the cloud. ThingSpeak provides instant visualizations of information or data posted by the peripheral devices. ThingSpeak fits in the cloud part and provides a platform to quickly data collection and analyze data from internet-connected sensor nodes. Some of the key features of ThingSpeak include the ability to [9]:

- Configure the application devices to send data to ThingSpeak Easily using internet protocol.

- Visualize the any sensor data on screen in real-time.

- Aggregate data on-demand from third-party sources.

- MATLAB is used to make sense the IoT data.

- IoT analytics based on schedules or events.

- Prototype and build IoT systems without developing web software.

\section{B. Virtuino}

Virtuino is a Human Machine Interface platform for IoT servers, Arduino ESP8266 or similar boards, MQTT brokers, and Modbus RTU bridge servers. The figure 5 shows the Virtuino Mobile App Screen. It allows creating amazing virtual screen on the tablet or phone to monitor and control the embedded system via Bluetooth, Wi-Fi or Web. It also supports to create visual interfaces with widgets like buttons, switches, LEDs, value displays, instruments, meters, etc. In this project, Virtuino Modbus is used which has an HMI for Modbus servers and supports ThingSpeak cloud server \& TCP/IP protocol.

\section{AIR POLLUTION MONITORING AND ITS IMPORTANCE}

Air quality monitoring is essential for major public and private industrial sectors to prevent air pollution and measure emission sources. It preserves the greenhouse effect. Industrial operators use monitoring equipment for air quality and manage emissions on their perimeter, which helps them to improve relationships with regulators and communities. With air quality regulation shifting the burden from publicly-funded monitoring to monitoring funded by industry, it has been increasingly important for businesses to acquire their quality monitoring equipment [10]. An air quality monitor is to measure the level of air pollutants is available in both indoor and outdoor ambient. Indoor air quality monitors are basically sensor-based devices. Some of them can measure ppb levels and it is portable units. The systems for air quality monitoring with data sharing are used widely in outdoor ambient applications. The impact of $\mathrm{RH}$ and $\mathrm{T}$ from the sensor response is tested by analyzing the relationship between observed PM2.5 sensor error (sensor observation minus reference data) and air temperature as well as $\mathrm{RH}$.

\section{A. Air Quality Index (AQI)}

An air quality index (AQI) is a number which is used by the government authorities to communicate the public about the current level of air pollution on a daily basis. It is a measure of air quality impacts their health. An increase in the AQI value tells that an increase in level of air pollution and the greater the severe adverse health effects. The concept of AQI is widely used in many countries in different point scales to report air quality [11].

\section{B. Setting-up Base Standard}

To measure the quality of air in the environment, we set the AQI category as a base standard of our system. It can identify the range of pollution and take necessary steps for future betterment. There are six AQI categories were given by the Ministry of Environment, Forest and Climate Change, Government of India is shown in table 1 [12]. The AQI values and categories as well as associated health impacts identifying faulty standards and environmental conditions.

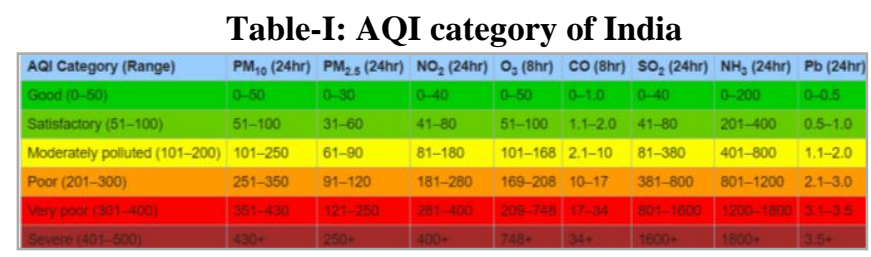

When the AQI range is between $0-50$ it is good and is shown in pure green color, when the AQI range is 51-100 it means that the data are satisfactory and are shown in greenish color. After that if the data range is 101-200 it will indicate that the area is moderately polluted and after that, if the range exceeds over 201 it means that the area has become polluted badly and when it goes to the range of 301-400, the AQI category becomes very poor and the area becomes highly polluted and unsafe for the human beings [13].

\section{RESULT ANALYSIS}

The concentration of pollutants in the air is measured in parts per million (ppm). The Analog Output pin of the sensor is connected to the analog channel of the MCU. The analog output from the MQ135 can be assumed directly proportional to the concentration (in ppm) of gas in the industrial area. The digitized value $\left(\mathrm{V}_{\text {out }}\right)$ is related to the load resistance to find the resistance output value from the sensor $\left(R_{s}\right)$.

$$
R_{\mathrm{s}}=\frac{V_{c} R_{L}}{V_{\text {out }}}-R_{L}
$$

where $V_{c}=5, R_{L}$ is load resistance and $R_{s}$ is the sensor resistance (between 30Kohm and 200Kohm). 
It is possible to obtain values of $\mathrm{CO}_{2}, \mathrm{CO}$ and $\mathrm{NH}_{4}$ levels in the air of the monitored environment using the following equations [14].

$$
\begin{aligned}
& \mathrm{CO}_{2}: \text { ppm }=114.3544 \times\left(\frac{R_{s}}{R_{0}}\right)^{-2.93599} \\
& \mathrm{CO}: p p m=662.9382 \times\left(\frac{R_{s}}{R_{0}}\right)^{-4.0241} \\
& \mathrm{NH}_{4}: p p m=102.694 \times\left(\frac{R_{s}}{R_{0}}\right)^{-2.48818}
\end{aligned}
$$

where $\mathrm{R}_{0}$ is the resistance of the gas sensor at defined concentration.

The ppm of $\mathrm{CO}_{2}$ gas in the environment varies from $4.6 \times 100 \mathrm{ppm}$ to $5.7 \times 100 \mathrm{ppm}$ which is shown in figure 5 . The safe upper limit for $\mathrm{CO}_{2}$ is no more than $450 \mathrm{ppm}$. The increasing $\mathrm{CO}_{2}$ concentration would lead to traps heat on the environment that can have a very significant effect on health. In order to stabilize $\mathrm{CO}_{2}$ concentrations less than $450 \mathrm{ppm}$, the microcontroller will turn on the exhaust fan.

The dust particles present inside the industry area are observed. The $\mathrm{PM}_{2.5}$ and $\mathrm{PM}_{10}$ values are compared with the reference signals during the calibration process. $\mathrm{PM}_{2.5}$ is able to enter deep into lung tissues and can cause various health issues [15]. It is also linked to temperature and humidity levels which affect the presence of pollutants in the air. Air quality monitors measure $\mathrm{PM}_{2.5}$ and $\mathrm{PM}_{10}$ concentrations in $\mu \mathrm{g} / \mathrm{m}^{3}$ for 24 hours. The conversion of air pollutants in parts per million to micrograms per cubic meters is given below,

Concentration $\left(\mathrm{mg} / \mathrm{m}^{3}\right)=$ concentration $(\mathrm{ppm}) \times \frac{\text { molecular mass }(\mathrm{g} / \mathrm{mol})}{\text { molar volume }(\mathrm{L})}$

The AQI provides an indication of the quality of the air and its health effects. The index value for pollutant can be calculated as [16]

$$
I_{p}=\frac{I_{H i}-I_{L o}}{B P_{H i}-B P_{L o}}\left(C_{P}-B P_{L o}\right)+I_{L o}
$$

where,

$\mathrm{C}_{\mathrm{p}}$ is the truncated concentration of pollutant (p)

$\mathrm{BP}_{\mathrm{Hi}}$ is the breakpoint that is greater than or equal to $\mathrm{C}_{\mathrm{p}}$

$\mathrm{BP}_{\mathrm{Lo}}$ is the breakpoint that is less than or equal to $\mathrm{C}_{\mathrm{p}}$

$\mathrm{I}_{\mathrm{Hi}}$ is the $\mathrm{AQI}$ value corresponding to $\mathrm{BP}_{\mathrm{Hi}}$

$\mathrm{I}_{\mathrm{Lo}}$ is the AQI value corresponding to $\mathrm{BP}_{\mathrm{Lo}}$

During the experiment, the device was placed in the industry area and monitoring the values for 24 hours. The results were recorded for every 5 minutes and respective graphical charts were plotted. The average value of AQI is 87 and 115 for $\mathrm{PM}_{2.5}$ and $\mathrm{PM}_{10}$ respectively. The air quality category for these AQI values is unhealthy for sensitive groups like people with respiratory disease.

\section{CONCLUSION}

Air pollution is one of the most dangerous hazards in many industries. It is necessary to monitor and ensure the workplace is healthy and free from pollutants. This IoT based air quality monitoring and control system using embedded system constantly maintain the air quality in the industrial workplaces and display the measured air quality index (AQI) on the ThingSpeak IoT platform and Virtuino mobile app display. It supports the new technology and effectively monitors environment parameters such as $\mathrm{CO}, \mathrm{CO}_{2}$, and ammonia gas concentrations, particulate matter $\mathrm{PM}_{2.5}$ and $\mathrm{PM}_{10}$ in air, and temperature-humidity in real-time. The data saved in the cloud server and also may send this information to the user via e-mail. If the threshold values for gas concentrations and PM are exceeded, the system has maintained the air quality index (AQI) in the workplace. This system will prevent the source of explosion and fire hazards due to gas leakages. The IoT based real-time air quality monitoring and control system to reduce the harmful effects in the industrial workplace.

\section{REFERENCES}

1. Shehab M.A, Pope F.D, Effects of short-term exposure to particulate matter air pollution on cognitive performance, Sci Rep 9 (2019) 8237. DOI:10.1038/s41598-019-44561-0

2. A. Sengupta, V. Varma, M. Sai Kiran, A. Johari, Marimuthu R, Cost-Effective Autonomous Garbage Collecting Robot System Using IoT And Sensor Fusion, International Journal of Innovative Technology and Exploring Engineering, 9 (2019) pp. 1-8.

3. Swati Dhingra, Rajasekhara Babu Madda, Amir H. Gandomi, Rizwan Patan, Mahmoud Dane, Internet of Things Mobile-Air Pollution Monitoring System (IoT-Mobair), IEEE Internet of Things Journal, 6 (2019) pp.5577-5584.

4. Xu Luo, Jun Yang, A Survey on Pollution Monitoring Using Sensor Networks in Environment Protection, Journal of Sensors 2019, Article ID 6271206.

5. Ghanem Osman Elhaj Abdalla, T. Veeramanikandasamy, Implementation of spy robot for a surveillance system using Internet protocol of Raspberry $\mathrm{Pi}, 2^{\text {nd }}$ IEEE International Conference on Recent Trends in Electronics, Information \& Communication Technology (2017) pp. 86-89. DOI:10.1109/RTEICT.2017.8256563.

6. K.Hemalatha, T.Srinivas, G.Swetha, V.Haripan, Effect of Air Quality Parameters in Hyderabad and Mapping Using QGIS and Detection Management Software, International Journal of Innovative Technology and Exploring Engineering, 9 (2019) pp. 73-80.

7. Kumar Keshamoni, Sabbani Hemanth, Smart Gas Level Monitoring, Booking \& Gas Lea-kage Detector over IoT, IEEE $7^{\text {th }}$ International Advance Computing Conference (IACC) (2017) pp. 330-332. DOI:10.1109/IACC.2017.0078.

8. Zeba Idrees, Zhuo Zou, Lirong Zheng, Edge Computing Based IoT Architecture for Low Cost Air Pollution Monitoring Systems: A Comprehensive System Analysis, Design Considerations \& Development, Sensors 18 (2018) 3021. DOI:10.3390/s18093021.

9. T. Veeramanikandasamy, R. Deepa, Detection of Trapped Victims in Disaster Scenarios Using IoT. ISBN-13: 978-1709421839.

10. Chen Xiaojun, Liu Xian-peng, Xu Peng, IoT-based air pollution monitoring and forecasting system, International Conference on Computer and Computational Sciences (ICCCS) (2015) pp. 257-260. DOI:10.1109/ICCACS.2015.7361361.

11. Harsh Gupta, Dhananjay Bhardwaj, Himanshu Agrawal, Vinay Anand Tikkiwal, Arun Kumar, An IoT Based Air Pollution Monitoring System for Smart Cities, IEEE International Conference on Sustainable Energy Technologies (2019) pp.173-177. DOI:10.1109/ICSETS.2019.8744949.

12. Scientific Evaluation of Air Quality Standards and Defining Air Quality Index for India.

13. Lutz Sager, Estimating the effect of air pollution on road safety using atmospheric temperature inversions, Journal of Environmental

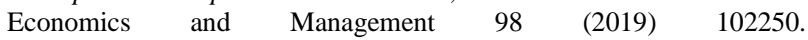
DOI:10.1016/j.jeem.2019.102250.

14. Robert Zalman, Veronika Olesnanikova, Peter Sevcik, Peter Sarafin, Monitoring of $\mathrm{CO}_{2}$ Amount in Closed Objects via WSN, Proceedings of the Federated Conference on Computer Science and Information Systems pp. 1257-1260. DOI: 10.15439/2015F332.

15. Sebastien Humbert, Julian D. Marshall, Shanna Shaked, Joseph V. Spadaro, Yurika Nishioka, Philipp Preiss, Thomas E. McKone, Arpad Horvath, and Olivier Jolliet, Intake Fraction for Particulate Matter: Recommendations for Life Cycle Impact Assessment, Environmental Science \& Technology 45 (2011) 4808-4816. DOI: 10.1021/es103563z. 
16. Hong Chen, Qiongsi Li MMath, Jay S Kaufman, Jun Wang, Ray Copes, Yushan Su, Tarik Benmarhnia, Effect of air quality alerts on human health: a regression discontinuity analysis in Toronto, Canada, The Lancet Planetary Health 2 (2018) e19-e26. DOI:10.1016/S2542-5196(17)30185-7.

\section{AUTHORS PROFILE}

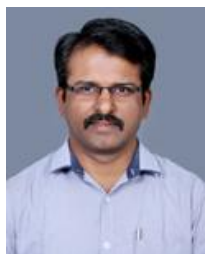

Dr. T. Veeramanikandasamy is an Assistant Professor of Electronics and Communication Systems at Sri Krishna Arts and Science College, Coimbatore 641008. He obtained his Doctorate in Electronics from Bharathiar University, Coimbatore, India. He has 13 years of teaching experience. His current research interests are in Nanomaterials Characterization, Embedded Systems, and Digital Signal Processing. Five candidates were awarded the M.Phil degree under his guidance. He has published three books with ISBN. He has published 11 research papers in peer-reviewed international journals. He has presented more than sixteen research papers in national/international conferences. He has delivered more than ten technical lectures in various institutions. He is a Life Member of the Indian Society of Systems for Science and Engineering (ISSE). He holds a certification in Embedded Software Engineer (NSQF-QP ELE/Q1501) received from the Electronics Sector Skills Council of India. He has developed various student enrichment courses on Embedded Systems, Digital System Design, Digital Signal Processing, Programmable Logic Controller, and IoT with Python.

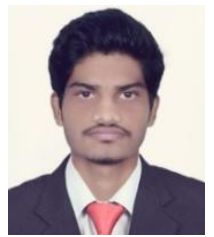

Gokulraj.S is studying under graduation in Electronics and Communication Systems at Sri Krishna Arts and Science College, Coimbatore 641 008. He got Best Paper Award in International Conference on Recent Advances in Communication, Energy and Sensor conducted by Sathyabama Institute of science and technology, Chennai. He is a Member of Institution Innovation Council of Sri Krishna Arts and Science College, Coimbatore 641008 during the year 2018-2019. He has attended the various National Level Workshops and Seminars. His areas of interest are embedded systems, Internet of Things, Digital Electronics and Programmable Logic Controller. He designed several embedded systems using assembly and Embedded C programming languages.

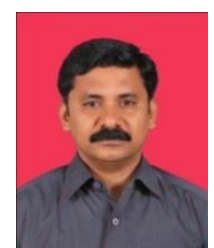

Dr. A. Balamurugan has 20 years of teaching experience at the undergraduate and postgraduate levels. He has obtained a Doctorate degree from Bharathiar University, Coimbatore, Tamilnadu, India. His fields of specialization include experimental nonlinear systems and nanomaterials. Under his supervision, five students were awarded Ph.D. and six students were awarded M.Phil degree. Currently, he is working as Professor and Head, Department of Physics, Government Arts and Science College, Avinashi, Tamilnadu, India. He also served on various committees of academic matter appointed by the State and Central Government. He has published more than thirty papers in national and international journals. He has presented more than sixty research articles in conferences. He has authored four books and acted as editor for publishing books. He has received the Best Researcher award.

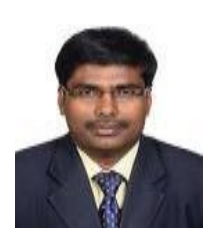

A.P. Ramesh received M.Sc and M.Phil degree in Applied Electronics from Bharathiar University, Coimbatore, India. He is currently working as an assistant professor in the Department of Electronics, Sri Ramakrishna College of Arts and Science, Coimbatore, India. His areas of interest include a real-time embedded system, digital electronics, electronics and communication systems and power electronics. He has 10 years of teaching experience and has published 5 research articles in international journals. He attended various national level seminars and hands-on trainings. He has presented more than 3 papers in conferences.

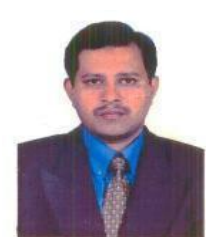

Dr. Y.A. Syed Khadar received B.E.S from Sengunthar Arts \& Science College, University of Madras, Chennai. M.Sc Applied Electronics from Bharathiar University, Coimbatore. M.Phil from Bharathidasan University, Tiruchirapalli and Ph.D in Electronics from Bharathiar University, Coimbatore in 2018. Currently he is working as an Associate Professor in the Department of Physics, KSR College of arts and science for women, Tiruchengode, Namakkal District. The author is a member in board of studies for Electronics in Periyar University, Salem. The author has more than 15 years of teaching experience and has published 15 research articles in national and international journals. His areas of interest are IoT, Microprocessor and microcontroller. 